\title{
Hybrid inversion method to estimate hydraulic transmissivity by combining multiple-point statistics and a direct inversion method
}

\author{
Alessandro Comunian* \\ Dipartimento di Scienze della Terra "A.Desio" \\ Università degli Studi di Milano, Milan, Italy \\ Mauro Giudici ${ }^{\dagger}$ \\ Dipartimento di Scienze della Terra "A.Desio" \\ Università degli Studi di Milano, Milan, Italy
}

This is the personal version of the manuscript published on Mathematical Geosciences, DOI: 10.1007/s11004-018-9727-0

\begin{abstract}
Inversion methods that rely on measurements of the hydraulic head $h$ cannot capture the fine scale variability of the hydraulic properties of an aquifer. This is particularly true for direct inversion methods, which have the further limitation of providing only deterministic results. On the other hand, stochastic simulation methods can reproduce the fine-scale heterogeneity but cannot directly incorporate information about the hydraulic gradient. In this work a hybrid approach is proposed to join a direct inversion method (the comparison model method, CMM) and multiple-point statistics (MPS), for determination of a hydraulic transmissivity field $T$ from a map of a reference hydraulic head $h^{(\mathrm{ref})}$ and a prior model of the heterogeneity (a training image). The hybrid approach was tested and compared with pure MPS and pure CMM approaches in a synthetic case study. Also, sensitivity analysis was performed to test the importance of the acceptance threshold $\delta$, a simulation parameter that allows one to tune the influence of $h^{(\mathrm{ref})}$ on the final results. The transmissivity fields $T$ obtained using the hybrid approach take into account information coming from the hydraulic gradient while simultaneously reproducing some of the fine scale features provided by the training image. Furthermore, many realizations of $T$ can be obtained thanks to the stochasticity of MPS. Nevertheless, it is not straightforward to exploit the correlation between the $T$ maps provided by the CMM and the prior model introduced by the training image, because the former depends on the boundary conditions and flow settings. Another drawback is the growing number of simulation
\end{abstract}

*e-mail: alessandro.comunian@unimi.it; corresponding author

${ }^{\dagger}$ e-mail: mauro.giudici@unimi.it 
parameters introduced when combining two diverse methods. At the same time, this growing complexity opens new possibilities that deserve further investigation.

\section{Introduction}

In hydrogeology, the problem of determining the hydraulic transmissivity field $T$ of an aquifer is often tackled using inversion methods that rely on information coming from the hydraulic head $h$. For this reason, many inversion methods cannot capture the fine-scale details of the hydraulic heterogeneity, because such information is filtered out by $h$ (Giudici and Vassena, 2008). This is particularly true for direct inversion methods, which strongly rely on estimates of $h$.

On the other hand, stochastic simulation methods, for example those that rely on some prior models of heterogeneity provided by two-point statistics (a variogram) or multiple-point statistics (a training image), cannot directly incorporate information coming from measurements of $h$.

Many authors have proposed approaches to address these two problems; their efforts were discussed and summarized in a recent review by Linde et al. (2015). Recent trends and the evolution of inverse methods in hydrogeology were also recently discussed by Zhou et al. (2014).

Carrera and Neuman (1986) were among the first to propose a successful inverse method, based on maximization of a likelihood function, the hypothesis of multi-Gaussianity of the data, and zonation. As a consequence of these assumptions, that method can hardly reproduce heterogeneity at small wavelengths. de Marsily et al. (1984) proposed the pilot points method (PiPM) to improve the fine-scale variability of the estimated parameter fields. Their method is based on the successive updating of a Kriging field. Some fictitious additional points (pilot points) are added to the points where $T$ was obtained with field tests, and Kriging is applied in an iterative fashion to improve the fit with the observations. The principle of the PiPM was then elaborated by Sahuquillo et al. (1992) and Gómez-Hernández et al. (1997) with the self-calibrated method (SCM). This method explores the advantages of inverse stochastic modeling by providing multiple realizations of the parameter fields under study, conditional to the state observations.

Both the PiPM and SCM rely on a prior model built upon two-point statistics. A number of authors have demonstrated the need for new and richer models of heterogeneity, beyond the restrictions of the variogram or multi-Gaussian assumption (e.g., Kerrou et al., 2008; Zinn and Harvey, 2003). An alternative to two-point statistics is to draw the prior information from training images, using multiple-point statistics (MPS, Strebelle, 2002; Mariethoz and Caers, 2014). This technique is among the promising tools for development of stochastic inverse modeling approaches (Zhou et al., 2014).

The works of Lochbühler et al. (2014), Laloy et al. (2016), Li et al. (2012, 2013, 2014), Ronayne et al. (2008), and Alcolea and Renard (2010) describe some recent developments and example applications of MPS within stochastic inversion frameworks. These works range from applications where conditioning data are based on geophysical measurements (Lochbühler et al., 2014), applications focused on data assimilation procedures based on ensemble an Kalman filter (EnKF, Li et al., 2012, 2013, 2014), applications integrating MPS with dy- 
namic data using the probability perturbation method in a Bayesian framework (Ronayne et al., 2008), and applications that take into account a connectivity constraint and head measurements with an acceptance/rejection criterion (Alcolea and Renard, 2010).

Notwithstanding this growing number of applications, there is still a need to explore the application of MPS within inversion frameworks; for instance, none of the above-mentioned works ever attempted to exploit the flexibility of MPS by combining it with a direct inverse method. This is done in this work, where MPS is combined with the comparison model method (CMM, Ponzini and Lozej, 1982). Despite the limitations of direct inversion methods (Zhou et al., 2014), the CMM has the advantage of being computationally parsimonious. The proposed hybrid method allows one to (i) include stochasticity in a deterministic inversion method, (ii) integrate MPS data related to the hydraulic head field and (iii) reproduce, at least partially, structures with relatively complex fine-scale structure.

Section 2 briefly recalls the working principles of the CMM and MPS and describes the workflow of the proposed hybrid inversion approach. It also describes an illustrative case study that will be used to test the proposed approach, and the comparison criteria adopted. The results obtained with a pure CMM, a pure MPS, and the proposed hybrid approach are reported in Sect. 3. Section 4 presents a discussion of the results, while Sect. 5 ends the manuscript with some concluding remarks.

\section{Methods}

The hybrid inversion method proposed herein is obtained by combining two techniques: one direct inversion method, i.e., the comparison model method (CMM, Ponzini and Lozej, 1982), and one geostatistical simulation algorithm, i.e., the direct sampling (DS, Mariethoz et al., 2010), which is part of a family of simulation methods based on multiple-point statistics (Guardiano and Srivastava, 1993; Strebelle, 2002). Note that, although two particular techniques are combined in this work, the proposed hybrid inversion workflow has a general validity; for example, DS could be replaced by another multiple-point simulation paradigm, for example snesim (Strebelle, 2002) or impala (Straubhaar et al., 2011), or any other geostatistical simulation technique that can integrate maps of auxiliary variables. DS is selected here due to its flexibility in handling auxiliary information and the possibility of reproducing connected and realistic patterns of heterogeneity.

In the following, the term "pure" is used in opposition to "hybrid" to describe the simulation approaches where either only MPS or only CMM is used.

\subsection{Comparison Model Method}

The comparison model method (CMM) is a direct inversion method useful for determination of a hydraulic transmissivity field $T$ given a source term $F$, a tentative initial transmissivity field $T_{k=0}^{(\mathrm{CM})}$ (the transmissivity of a comparison model, CM), some boundary conditions (BCs), and a reference hydraulic head field $h^{(\text {ref })}$ (Scarascia and Ponzini, 1972; Ponzini and Lozej, 1982; Ponzini and 
Crosta, 1988). The latter is very often interpolated from sparse measurements of the hydraulic head $h$ over the domain to be characterized.

Figure 1 illustrates the working principle of the CMM as a flowchart. The main idea is to update iteratively an initial field $T_{k=0}^{(\mathrm{CM})}$. At each iteration $k$, a forward problem (FP) is solved for a $\mathrm{CM}$ that shares the same source terms and boundary conditions as the real problem. Using a tentative transmissivity field $T_{k}^{(\mathrm{CM})}$, a head field $h_{k}^{(\mathrm{CM})}$ is obtained. The basic hypothesis applied to update the transmissivity field is that the hydraulic fluxes computed for the CM should be very close to the fluxes related to $h^{(\mathrm{ref})}$. In other worlds, the update step can be expressed as

$$
T_{k+1}^{(\mathrm{CM})}=T_{k}^{(\mathrm{CM})} \frac{\left|\nabla h_{k}^{(\mathrm{CM})}\right|}{\left|\nabla h^{(\mathrm{ref})}\right|},
$$

where the hydraulic gradients $\nabla h$ can be computed using a finite-difference scheme in practical applications. In this way, $T^{(\mathrm{CM})}$ is updated iteratively until a stopping criterion is met. At the end of this procedure, $T_{k+1}^{(\mathrm{CM})}$ is taken as the estimated $T$ field $T^{(\text {est })}$.

The CMM has been successfully applied in a number of case studies (e.g., Vassena et al., 2012; De Filippis et al., 2016), and beyond the field of hydrogeology (Lesnic, 2010). One of its strengths is computational efficiency, because at each iteration, one only has to solve a FP, and few iterations are needed to obtain a reliable $T^{(\text {est })}$ in most cases. Another strength is the possibility of obtaining a transmissivity field representative of the model scale, i.e., of the spacing of the chosen estimation grid.

Among the weaknesses of the CMM is the difficulty in reproducing the finescale details of a $T$ field. This arises from the fact that $h^{(\mathrm{ref})}$ filters out the fine-scale features of the underlying parameter field $T$. Therefore, the aim of this work is to address this weakness by injecting some a priori information using multiple-point statistics simulation (Guardiano and Srivastava, 1993; Strebelle, 2002).

\subsection{Direct Sampling Method (DS)}

As for other multiple-point simulation paradigms, DS is based on the concept of a training image (TI), which represents a conceptual model of the heterogeneity and serves as a database of patterns. Here, the working principle of this technique is briefly outlined; More details can be found in Mariethoz et al. (2010).

The aim of DS is to fill the gaps in the spatial variability of a random variable $Z$ defined only at limited locations in a simulation grid (SG), by sampling the spatial patterns provided by a TI. The sequential DS simulation procedure, illustrated in Fig. 2, can be summarized as follows:

1. Given a search radius $R$, randomly select an empty cell at location $\mathbf{x}$ in the SG. The cells of the SG where a value of $Z$ is already defined (or simulated) and which fall within a radius $R$ around $\mathbf{x}$ are then selected. Their values, together with their location in the SG with respect to $\mathbf{x}$ (the lag vectors $\left.\mathbf{l}_{1}, \mathbf{l}_{2}, \ldots\right)$, define a data event $e=\left\{Z\left(\mathbf{x}+\mathbf{l}_{1}\right), Z\left(\mathbf{x}+\mathbf{l}_{2}\right), \ldots\right\}$ (Fig. 2a). 


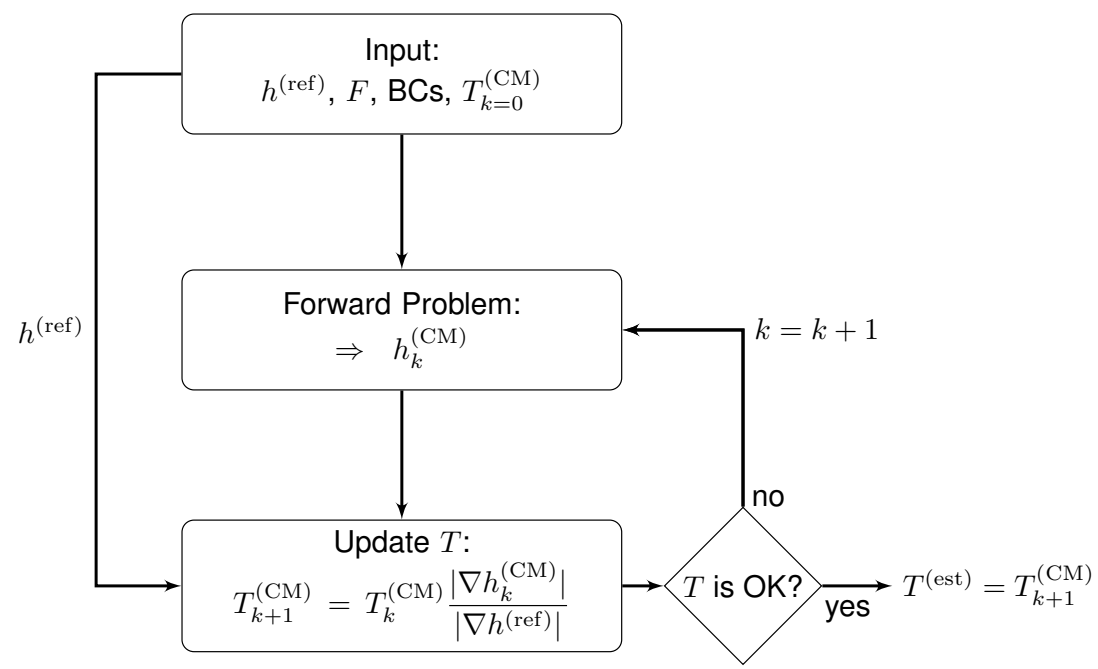

Figure 1: Flowchart of CMM method

2. The TI is scanned for a data event similar to the data event $e$ defined in the previous step for the SG. Different measures of similarity can be adopted to define the distance $d$ between two data events. In the case of categorical variables, one measure can be based on the number of cells that present the same value of $Z$. In the case of continuous variables, other options are available. Here, the $\mathcal{L}^{1}$-norm is considered.

3. Given a user-defined threshold value $\delta$, if $d<\delta$ then the similarity between the two data events is "accepted" and the value at the center of the data event in the TI is pasted at the corresponding grid location $\mathbf{x}$ in the SG (Fig. 2c). Otherwise (Fig. 2b), the search continues up to a given stopping criterion.

These steps are repeated sequentially to fill all the gaps in the SG. In addition, at the beginning of this procedure, the variable $Z$ is normalized in order to work with dimensionless quantities for $d$ and $\delta$.

The aforementioned procedure can be easily extended to the case of a multivariate random variable $\mathbf{Z}$ by providing a multivariate TI, a vector threshold $\delta$, and a distance for each component of $\mathbf{Z}$; For example, concerning the choice of this distance, $\mathbf{Z}$ could be composed of a categorical variable and a continuous variable. Therefore, one could use a dedicated distance for the categorical component of $\mathbf{Z}$ and a different distance for its continuous component. Based on this flexibility, it is easy to imagine a collocated simulation framework, where a bivariate TI is provided while on the SG only one variable is exhaustively known and the other must be simulated. Indeed, this is the setting of the proposed hybrid method: (a) a bi-variate TI, containing a categorical representation of $T$ in terms of facies codes (channel and background) and the corresponding continuous image obtained with the CMM, and (b) a SG where only the continuous estimate obtained by the CMM is provided, and the categorical representation must be simulated. 
a)

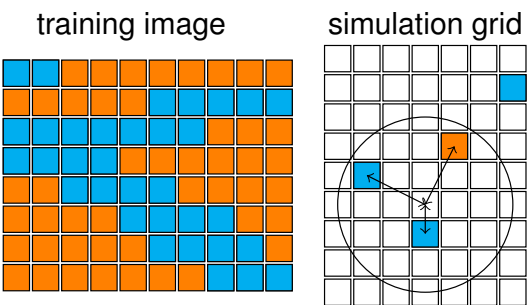

b)
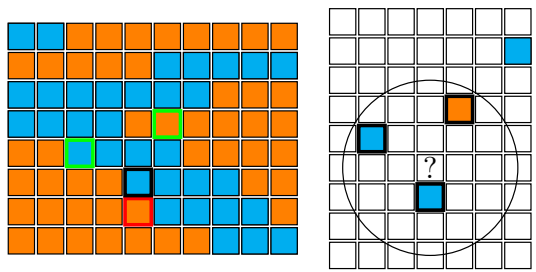

c)
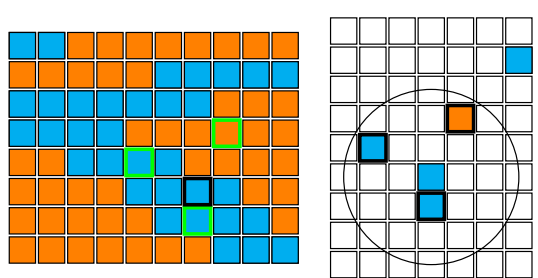

Figure 2: Three basic steps in the DS simulation procedure: a) selection of a random location and a data event in the SG; b) selection of a data event in the TI and compatibility check; c) a compatible data event is found in the TI, and its center value is pasted in the SG 


\subsection{Hybrid Inversion Method}

The proposed hybrid inversion workflow is composed of one preliminary step and one main step. The preliminary step consists in application of the pure CMM approach to create a series of multivariate TIs, one for each iteration of the CMM. The $h^{(\mathrm{ref})}$ used for the CMM is obtained by solving a FP with a training transmissivity field $T^{(\mathrm{TI})}$ representing an a priori model of heterogeneity that is expected to contain the same patterns of heterogeneity as the unknown $T$ field. $T^{(\mathrm{TI})}$ can be categorical, with a $T$ value provided for each category, or continuous. In this preliminary step, the pure CMM is run as depicted in Sect. 2.1 for a given number $K$ of iterations with given BCs and source term $F$. At the end of the $K$ CMM iterations, $K$ transmissivity fields $T_{k=1}^{(\mathrm{TI}, \mathrm{CM})}, \ldots$, $T_{k=K}^{(\mathrm{TI}, \mathrm{CM})}$ are obtained. These fields, combined with $T^{(\mathrm{TI})}$, represent a number $K$ of bivariate training images $\left(T^{(\mathrm{TI})}, T_{k=1}^{(\mathrm{TI}, \mathrm{CM})}\right), \ldots,\left(T^{(\mathrm{TI})}, T_{k=K}^{(\mathrm{TI}, \mathrm{CM})}\right)$ that are used in the following step.

Figure 3 illustrates the main step. In this step, the $h^{(\text {ref })}$ used in the CMM steps is not, in general, computed by solving a FP on a fully known $T$ field, as it is in the first step. Instead, here $h^{(\mathrm{ref})}$ could be, for example, the result of an interpolation of some $h$ measurements over an unknown $T$ field that is to be estimated. Then, the hybrid CMM is applied updating $T$ in two subsequent steps. First, $T_{k}^{(\mathrm{CM})}$ is updated using the information provided by $\nabla h_{k}^{(\mathrm{CM})}$ and $\nabla h^{(\mathrm{ref})}$, as for the pure CMM, using Eq. (1). Second, the obtained $T_{k+1}^{(\mathrm{CM})}$ is used as a collocated variable to simulate, using DS, a newly updated $T$. For the DS simulation, in addition to the $T_{k+1}^{(\mathrm{CM})}$ used as a collocated variable, the bivariate TI computed at the previous step for the corresponding $k$ is used, i.e., $\left(T^{\mathrm{TI}}, T_{k+1}^{(\mathrm{TI}, \mathrm{CM})}\right)$. As for the pure $\mathrm{CMM}$, this procedure is repeated until a stopping criterion for the estimated $T$ is met.

This hybrid inversion framework was implemented using the parflow (Ashby and Falgout, 1996; Jones and Woodward, 2001; Kollet and Maxwell, 2006; Maxwell, 2013) and YAGMod (Cattaneo et al., 2015) simulation platforms for solution of all the FPs, the deesse software (Mariethoz et al., 2010) for the DS simulation, and Python and its numerical libraries for the CMM and remaining numerical tasks.

\subsection{Illustrative Case Study}

To illustrate the diverse behaviors of the pure MPS, the pure CMM, and the proposed hybrid approach, the starting point is the TI proposed by Strebelle (2002). A target heterogeneity akin to that TI represents a challenge for all inversion techniques that rely on the two-point correlation paradigm, and would also be a challenge for the pure CMM.

Using the aforementioned TI in a DS simulation framework, two realizations on a grid of $300 \times 200$ square cells of side $25 \mathrm{~m}$ were obtained using the deesse DS simulation code (Mariethoz et al., 2010). One of the two realizations was used in the following as a training image itself $\left(T^{(\mathrm{TI})}\right.$, Fig.4a), while the other was considered as the (supposed unknown) target transmissivity field that one wishes to reproduce $\left(T^{\text {(true) }}\right.$, Fig. 4b). Using average values for sand and shale extracted from the worldwide hydrolgeological parameters database (http://wwhypda.org, Comunian and Renard, 2009), and considering an aquifer of thickness $10 \mathrm{~m}$, 


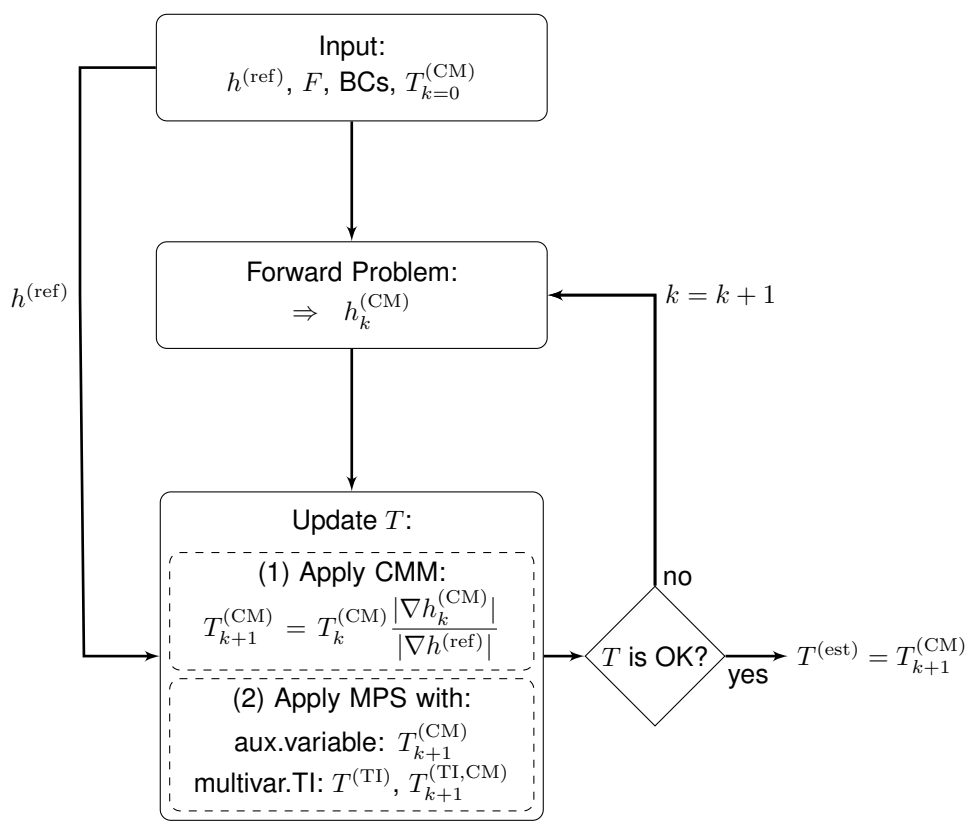

Figure 3: Flowchart for hybrid inversion approach

transmissivity values of $1 \times 10^{-3} \mathrm{~m}^{2} / \mathrm{s}$ and $1 \times 10^{-6} \mathrm{~m}^{2} / \mathrm{s}$ were assigned to the sand and background shale facies, respectively. In the following, the terminology "low-transmissivity/high-transmissivity" or "background/channel" cells is used interchangeably. In all the simulation settings involving the CMM, the method was applied considering a null source term $F$ and fixed head boundary conditions at the borders corresponding to the cell indices $j=1(h=10.0 \mathrm{~m})$ and $j=$ $300(h=9.0 \mathrm{~m})$, to produce a hydraulic gradient along the main direction of orientation of the channels, with null flux elsewhere.

Figure $4 \mathrm{c}$ shows the head field $h^{\text {(true) }}$ obtained from the solution of the forward problem for the reference $T$ field; in this map, the yellow dots show the randomly selected positions of the 30 virtual monitoring points, i.e., the nodes where it is assumed that $h^{(\text {true })}$ is measured. These data will be compared with the values computed by solving the forward problem for other estimated $T$ fields. Note that these monitoring points are used only for statistics on estimated heads, but not as conditional points for transmissivity or facies.

\subsection{Comparison Criteria}

The following parameters were used to evaluate the performance of the different techniques: The proportion of correctly simulated channels $\left(p_{\text {rgt }}\right)$ quantifies the proportion of simulated channel cells that correspond to a channel in the $T^{\text {(true) }}$ grid, while the proportion of incorrectly simulated channels $\left(p_{\mathrm{wrg}}\right)$ is the proportion of simulated channel cells that do not correspond to a channel in the $T^{\text {(true) }}$ grid. The Jaccard index $(J)$ somehow summarizes these two parameters $p_{\text {rgt }}$ and $p_{\mathrm{wrg}}$. In terms of sets, if $A$ represents the set of the simulated channel facies and $B$ the set of the channel facies in the reference, then the Jaccard 
index is defined as

$$
J=\frac{|A \cap B|}{|A \cup B|} .
$$

Another parameter considered herein is the proportion of channel facies $(p)$, that is, the number of channel facies over the total number of grid cells. It is also useful to examine the probability of connection of two points $\mathbf{x}$ and $\mathbf{y}$, i.e., the probability that two points belong to the same connected component (or geobody) of the considered domain; this is done using the intrinsic $\left(c_{\mathrm{int}}\right)$ and total connectivity $\left(c_{\text {tot }}\right)$, as defined by Vassena et al. (2010). These parameters are related to the hydraulic properties of porous media and therefore provide a synthetic way to summarize them. Here, in particular, the connectivity of the more conductive cells (channel facies) is considered using a four-point scheme. In other words, the connectivity is considered only through the sides of the cells but not through their corners. (See Vassena et al. (2010) for a formal definition, and Renard and Allard (2013) for a comprehensive and recent review about the concept of connectivity and different indicators). Another important morphometric attribute that is useful for comparison of different realizations of a categorical variable is the number of clusters $(N)$, i.e., the number of connected components. Here, again, the number of connected components for the channel facies is considered.

All these parameters were computed both on the whole simulation domain and on a subdomain obtained by excluding a boundary frame of size 50 cells on each side. This subdomain is useful to reduce the influence of the boundary conditions on the results of the simulation (an influence that will become evident in the next section in the pure CMM results).

In this work, a synthetic case study is examined, to enable access to a known transmissivity field $T^{\text {(true) }}$ (Fig. 4b). Therefore, in addition to the aforementioned parameters, the following statistical indicators are adopted to quantify the errors in the computation of the estimated transmissivity field $T$ :

$$
\begin{aligned}
\lambda & =\frac{1}{M} \sum_{i=1}^{M}\left(\log _{10}\left(T^{\text {(true })}(i)\right)-\log _{10}\left(T^{(\text {est })}(i)\right)\right), \\
|\lambda| & =\frac{1}{M} \sum_{i=1}^{M}\left|\log _{10}\left(T^{\text {(true })}(i)\right)-\log _{10}\left(T^{(\text {est })}(i)\right)\right|, \\
\lambda^{2} & =\frac{1}{M} \sum_{i=1}^{M}\left(\log _{10}\left(T^{(\text {true })}(i)\right)-\log _{10}\left(T^{(\text {est })}(i)\right)\right)^{2},
\end{aligned}
$$

where $M$ is the number of nodes at which the transmissivity is estimated, and $T^{\text {(true) }}(i)$ and $T^{(\text {est) }}(i)$ are the transmissivity values at cell $i$, for the reference and estimated fields, respectively. Note that $\lambda,|\lambda|$, and $\lambda^{2}$ are dimensionless parameters, because they are based on logarithms of transmissivity ratios. In addition, to quantify the impact that the estimates of $T$ have on the hydraulic head $h$, a root-mean-squared error (RMSE) was computed using the values of $h^{\text {(true) }}$ at the virtual monitoring points (Fig. 4c) and the values of $h$ computed using the results of the pure CMM $\left(h^{(\mathrm{CM})}\right)$. 


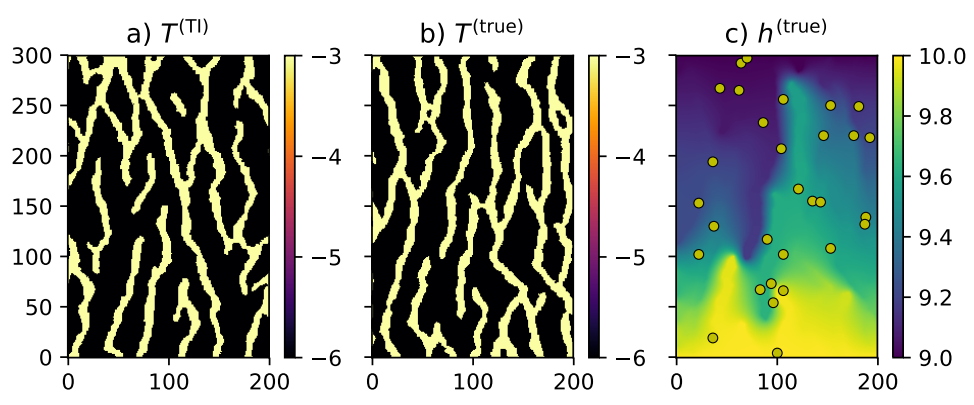

Figure 4: Decimal logarithm of the $T$ field used as a) training image and as b) reference. Measurement units are $\mathrm{m}^{2} / \mathrm{s}$ for $T$ and $\mathrm{m}$ for $h$. Note that, in this figure and in all the subsequent figures containing maps, values on axes refer to cell indexes

\section{Results}

In this section, the results obtained using a pure MPS approach, a pure CMM approach, and the proposed hybrid approach are illustrated.

\subsection{Pure MPS}

To illustrate the differences between the proposed hybrid methodology and a standard approach, the considered reference is an ensemble of 10 pure MPS realizations performed using different random seeds with standard set of simulation parameters and the TI presented in Fig. 4a. The parameter sets were selected according to the guidelines suggested by Meerschman et al. (2013). In particular, a search radius of 124 cells, a maximum number of neighboring cells of 24 , and a threshold $\delta=0.05$ were selected for scanning the $30 \%$ of the training image. These same parameter values will be used below in the hybrid approach, apart from relatively small differences required to include a secondary variable. For the moment, in the application of the pure MPS approach, neither hard conditioning data nor soft conditioning data such as auxiliary variable maps were used in the simulations illustrated in this section. The aim here is to evaluate the default performance of the pure MPS simulation method when trying to reproduce the reference $T$ field presented in Fig. 4b.

The results of application of the pure MPS approach are illustrated in Fig. 5. A boxplot is drawn for each of the parameters introduced in the previous sections, viz. $p, p_{\mathrm{rgt}}, p_{\mathrm{wrg}}, c_{\mathrm{int}}, c_{\mathrm{tot}}, J$, and $N$, for the full domain and subdomain.

\subsection{Pure CMM}

This work proposes a hybrid inversion technique that makes use of a direct inversion method and a geostatistical technique. It is therefore useful to briefly present the results that could be obtained using the direct inversion method only, to highlight the improvements introduced when using the proposed hybrid technique.

In a real case study, it is of course not possible to take measurements of the hydraulic head with the same spatial density as the considered discretization grid. Nevertheless, here the results that could be obtained using the pure CMM 
a)

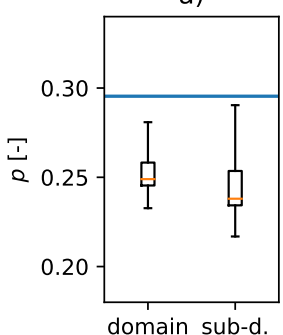

e)

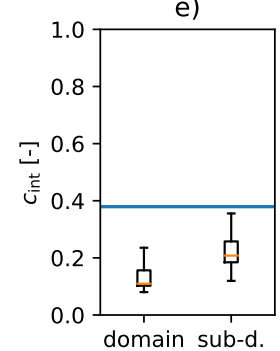

b)

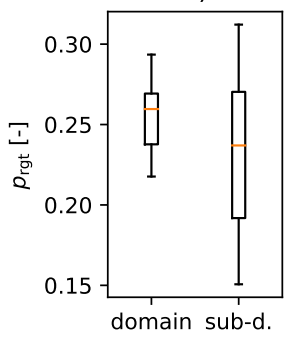

f)

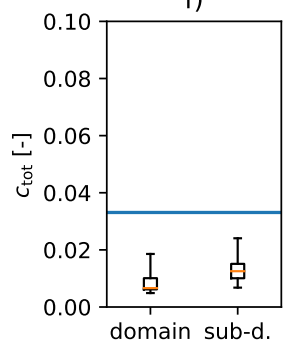

c)

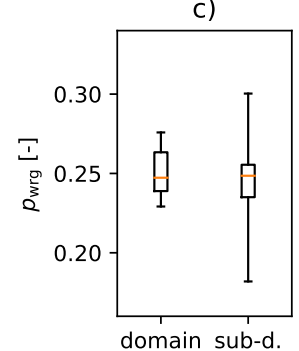

g)

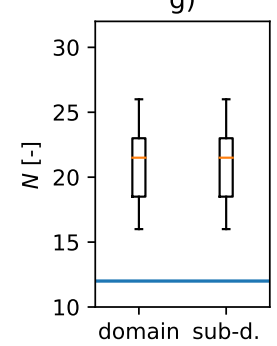

d)

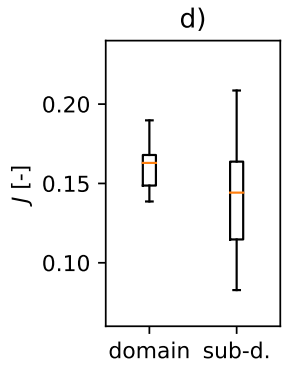

Figure 5: Reference parameters for pure MPS simulation, computed on full domain and on subdomain (neglecting an external frame of 50 cells). Horizontal lines represent parameters computed on the reference field (Fig. 4b)

when $h$ is known at every point of the discretization grid are briefly outlined. In particular, in Fig. $6, \lambda,|\lambda|, \lambda^{2}$, and $h^{(\mathrm{RMSE})}$ are plotted against the number of CMM iterations for the full domain (cyan continuous line) and subdomain (orange dashed line). Figure 7 shows maps of $h^{\text {(true) }}, h^{(\text {ref })}, h^{(\mathrm{CM})}, \log _{10}\left(T^{(\text {true })}\right)$, $\log _{10}\left(T^{(\mathrm{CM})}\right)$, and $\left(h^{(\text {true })}-h^{(\mathrm{CM})}\right)$ for the third CMM iteration. This iteration was chosen to show and discuss the results, because $\lambda^{2}$ attains a local minimum for the reduced domain, $h^{(\mathrm{RMSE})}$ shows a small variation with respect to the preceding iteration, and the trend of $|\lambda|$ becomes less negative for subsequent iterations.

\subsection{Hybrid Approach}

As explained in Sect. 2.3, a preliminary step is required before running the hybrid approach. This step is needed to create the multivariate training images that will be used for the multivariate DS simulations within the workflow of the hybrid approach. These multivariate TIs are obtained by running a pure CMM approach using a $h^{(\mathrm{ref})}$ computed by solving a FP on the $T$ field defined by the TI in Fig. 4a. The $T$ maps created by the pure CMM for each iteration are used in the multivariate TIs as secondary variable, while the main variable (which is the same for all iterations) is the TI in Fig. 4a. Since within the hybrid approach the DS is applied with a multivariate TI, a vectorial threshold $\delta=\left(\delta_{1}, \delta_{2}\right)$ is required. The first component $\left(\delta_{1}\right)$ controls the acceptance threshold for the categorical variable channel/background, while the second component $\left(\delta_{2}\right)$ controls the threshold acceptance for the continuous variable simulated with the CMM.

The hybrid approach was first tested using a true $h^{(\text {ref })}$ and $\delta=(0.01,0.001)$. 
a)

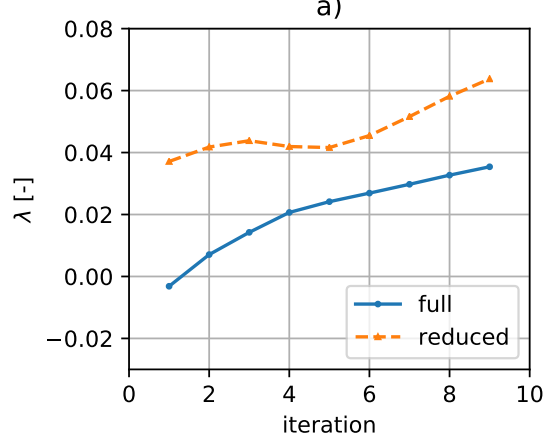

c)

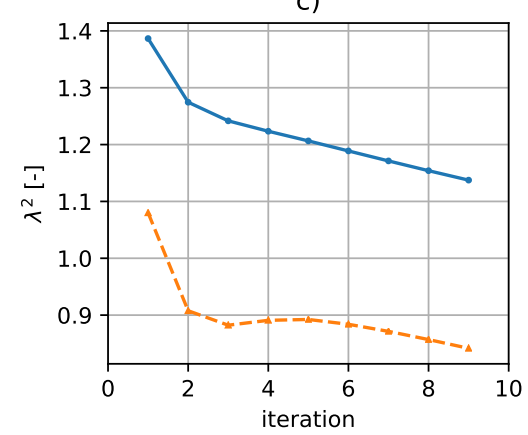

b)

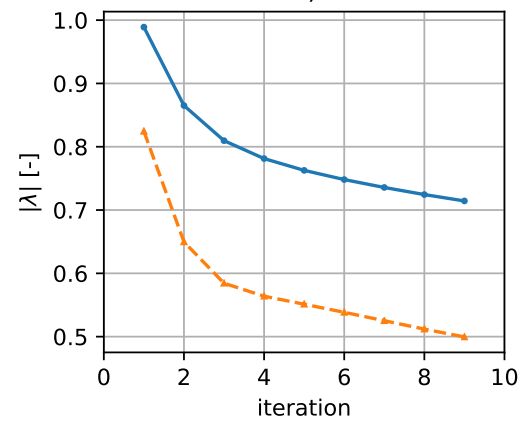

d)

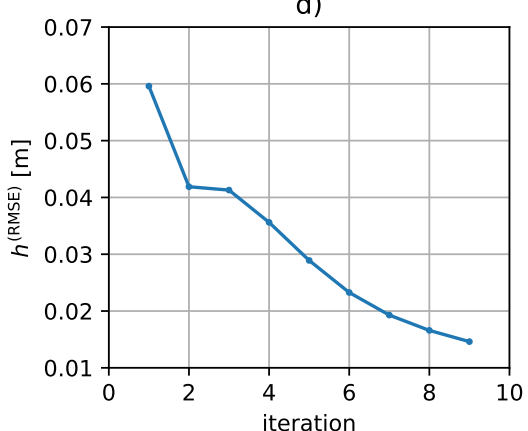

Figure 6: Performance of pure CMM in terms of $\lambda,|\lambda|, \lambda^{2}$ and $h^{(\mathrm{RMSE})}$ when $h$ is known in all cells of the simulation domain. a)-c) present the trends for the full simulation domain (cyan continuous line) and subdomain (orange dashed line) 


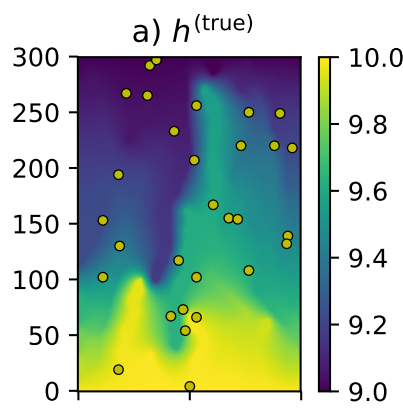

d) $T^{\text {(true) }}$

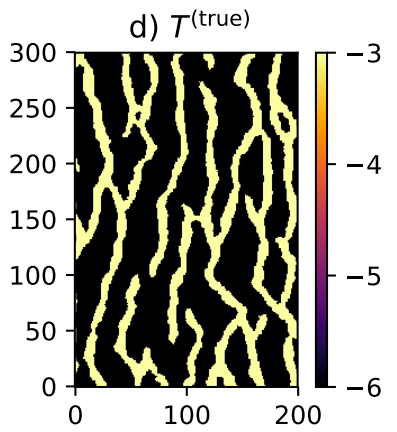

b) $h^{\text {(ref) }}$

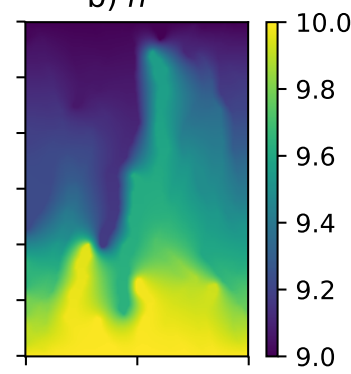

e) $T_{k=3}^{(\mathrm{CM})}$

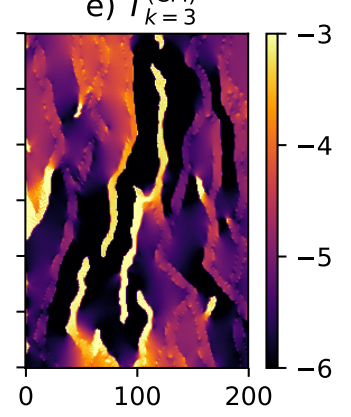

c) $h_{k=3}^{(\mathrm{CM})}$

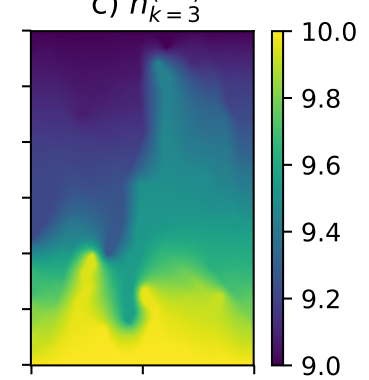

f) $h^{\text {(true) }}-h_{k=3}^{\text {(CM) }}$

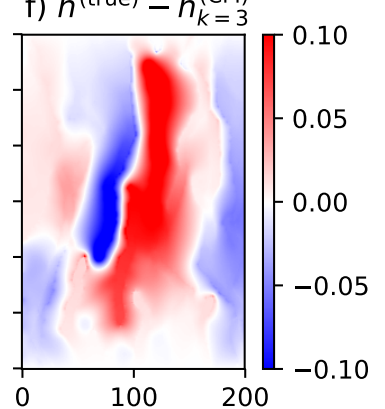

Figure 7: Results of CMM inversion after three iterations: a) true $h$ field (yellow dots represent locations used to compute $\left.h^{(\mathrm{RMSE})}\right)$, b) reference $h$ used as input for the CMM (in this case $h^{(\text {ref })}=h^{\text {(true) }}$ ), c) $h$ computed with the $T$ field estimated with the CMM, d) logarithm of the reference $T$ field, e) logarithm of the $T$ field estimated with the CMM, f) $h^{(\text {true })}-h^{(\mathrm{CM})}$. Units are $\mathrm{m}$ for hydraulic head, and $\mathrm{m}^{2} / \mathrm{s}$ for the transmissivity 
Then, always using a true $h^{(\text {ref })}$, a sensitivity test was performed on $\delta_{2}$ only.

\subsubsection{True $h$}

In this test, the hybrid approach was applied using $h^{(\text {ref })}=h^{\text {(true) }}$ as input, i.e., an $h$ field obtained by solving a FP using the true $T$ (Fig. 4b). This setting is of course far from the real case situation; still, it is useful to verify the performance of the proposed hybrid approach in an ideal case.

Figure 8 shows the same maps presented in Fig. 7 for the pure CMM, with three additional maps: g) the binary component of the multivariate TI image (Fig. 4a), h) the continuous component of the multivariate TI, obtained in the preliminary step by running the pure CMM, and i) the binary result of the hybrid inversion approach.

A crucial parameter that controls the quality of the DS simulation is the threshold value $\delta$ used to accept/reject a given data event (Meerschman et al., 2013). For this example, $\delta_{1}$ and $\delta_{2}$ were fixed at 0.01 and 0.001 , respectively. The next section presents a brief sensitivity analysis on $\delta_{2}$.

\subsubsection{Sensitivity Analysis on the Threshold $\delta_{2}$ for the Secondary Variable}

As anticipated in Sect. 3.3.1, the acceptance threshold $\delta$ plays an important role in the results of the DS simulations. In this example, the effects of variation of this parameter $\left(\delta_{2}\right)$ are illustrated for the secondary variable, i.e., $T^{(\mathrm{CM})}$. A different value of $\delta_{2}$ corresponds to giving a different weight to the information provided by $h^{(\text {ref })}$ in the hybrid approach. To illustrate the effects of changing $\delta_{2}$, the results of the first 10 iterations of the hybrid approach were stacked and an average value computed and mapped for $\delta_{2}=0.001, \delta_{2}=0.1, \delta_{2}=0.2$, and $\delta_{2}=0.5$ (Fig. 10). It is also useful to compare the results obtained using different values of $\delta_{2}$ in quantitative terms. Figures 11 and 12 show boxplots of $N$ and $J$ obtained by running 10 simulations with the hybrid approach with different values of $\delta_{2}$. The first boxplot represents $N$ and $J$ for the pure MPS approach, while the others correspond to different $k$-th iteration steps of the hybrid approach.

\subsection{CPU Time}

Table 1 reports the CPU time required to run the three methods. For the times related to the pure CMM and the hybrid approach, all the iterations required to obtain an acceptable result (in terms of the aforementioned parameters) are considered. These results were obtained by running the case study on a workstation equipped with an Intel ${ }^{\circledR}$ Core $^{\mathrm{TM}}$ i7 CPU 860 (2.80 GHz clock frequency) using one core only. However, all the reported times could be further reduced because both parflow and deesse can be run using multiple cores. Note that, for the hybrid approach, about $98 \%$ of the CPU time is spent on the MPS simulations, while the remaining $2 \%$ is used for solving the FP and by the Python utility script. Notice also that the MPS iteration performed within the hybrid approach is expected to be slower than the pure MPS, because for the hybrid approach two variables are considered. 
a) $h^{\text {(true) }}$

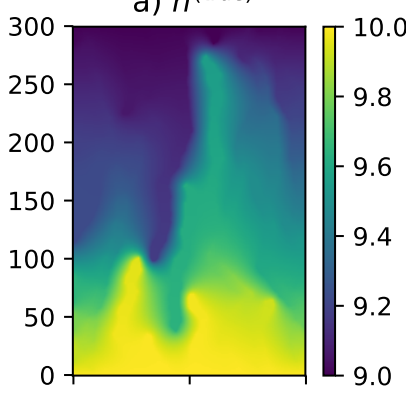

d) $T^{\text {(true) }}$

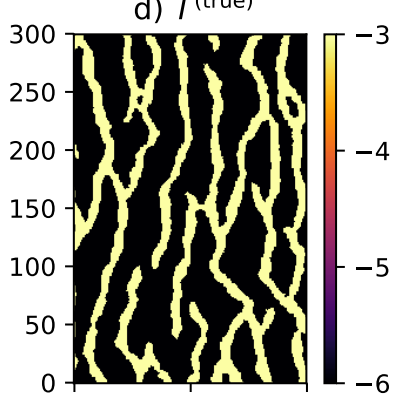

g) $T^{(\mathrm{TI})}$

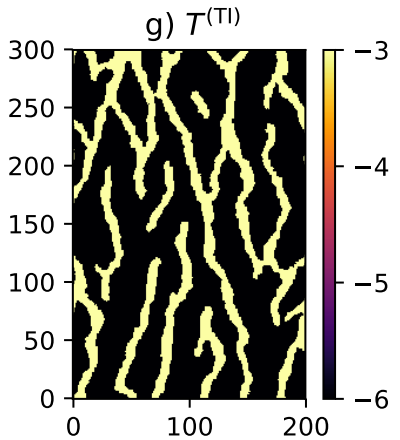

b) $h^{\text {(ref) }}$

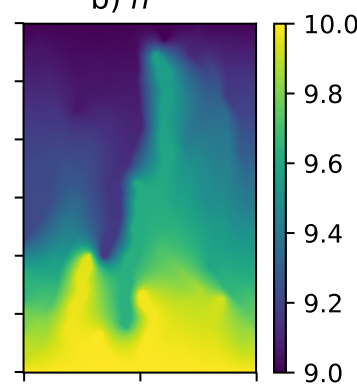

e) $T_{k=1}^{(\mathrm{CMM})}$

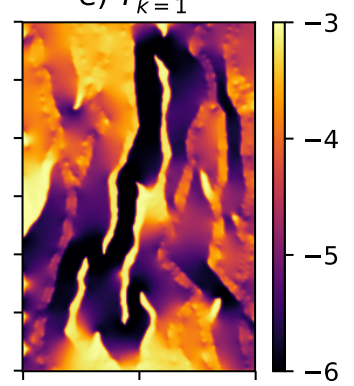

h) $T_{k=1}^{(\mathrm{TI}, \mathrm{CMM})}$

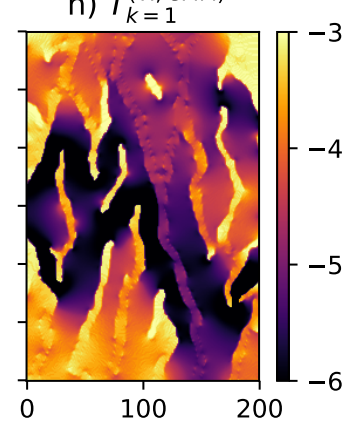

c) $h_{k=1}^{\text {(CMM) }}$

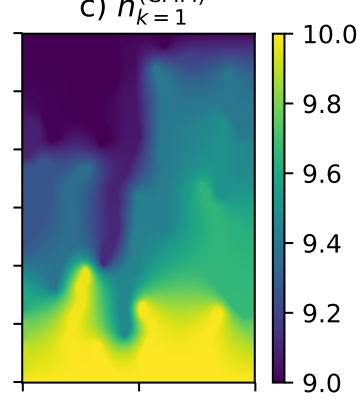

f) $\left(h^{\text {(true) }}-h_{k=1}^{\text {(CMM) }}\right)$
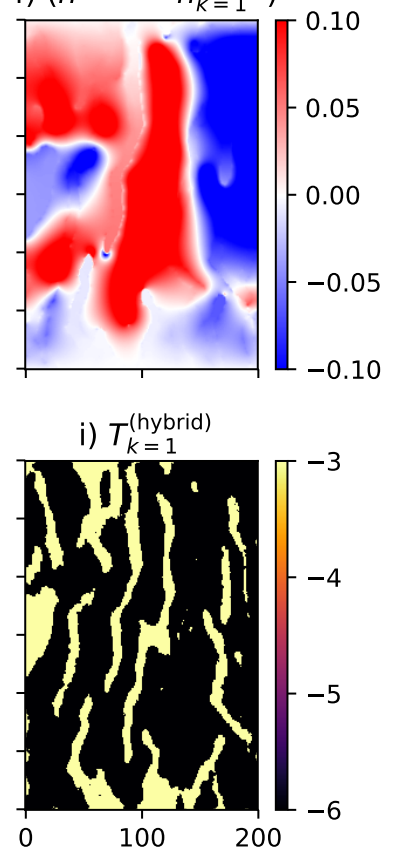

Figure 8: Results of hybrid approach using $h^{(\text {ref })}=h^{(\text {true })}$ and $\left(\delta_{1}, \delta_{2}\right)=$ (0.01,0.001), first iteration: from a) to f) see Fig. $7, \mathrm{~g}) \operatorname{logarithm}$ of $T$ field used as categorical training image $\mathrm{h}$ ) secondary continuous variable used as training image, i.e., the logarithm of the $T$ computed using the pure CMM in the preliminary step, i) result of application of hybrid approach

Table 1: CPU time required by the three methods. The number of iterations is meaningful only for the pure CMM and CMM+MPS, corresponding to the number of iterations needed to obtain an acceptable $T$ field.

\begin{tabular}{lcr}
\hline method & No. iter. & CPU time [sec] \\
\hline Pure MPS & - & 58.2 \\
Pure CMM & 3 & 4.3 \\
Hybrid (CMM+MPS) & 1 & 160.1 \\
\hline
\end{tabular}


a) proportions (facies 1 )

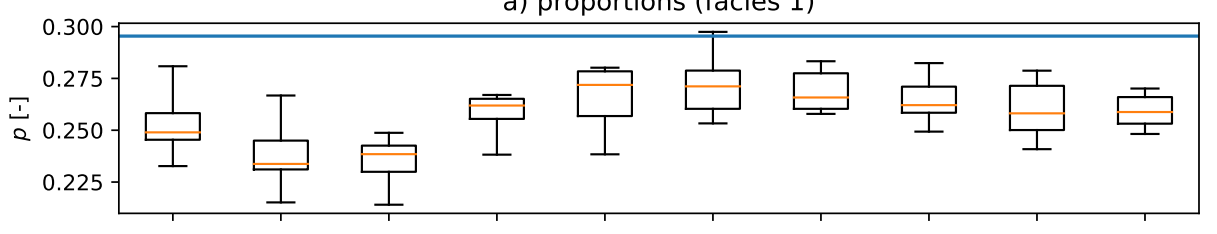

b) right simulated channels

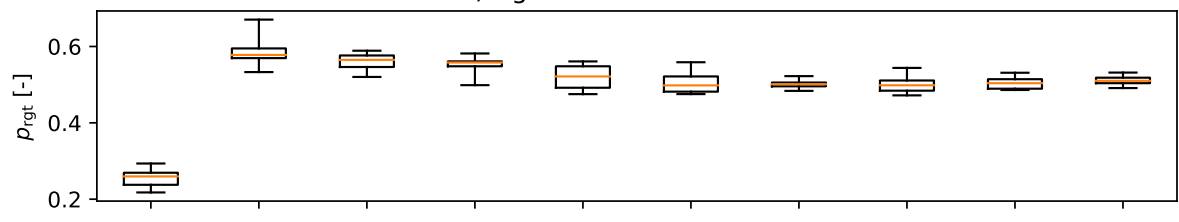

c) wrong simulated channel

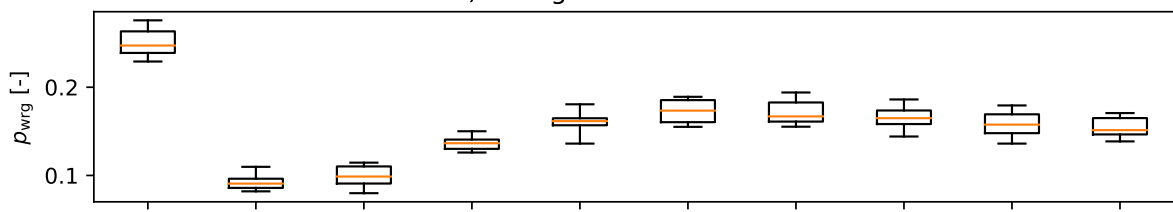

d) intrinsic connectivity

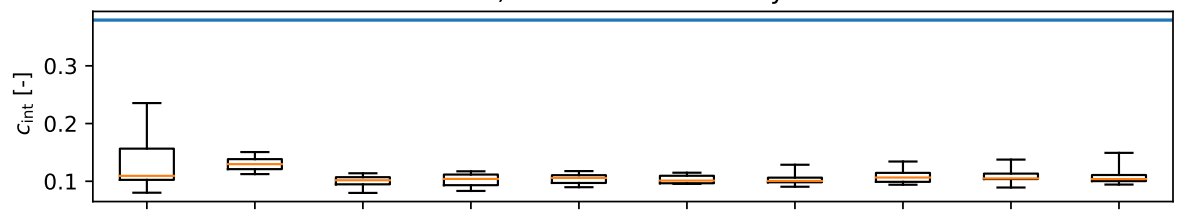

e) total connectivity
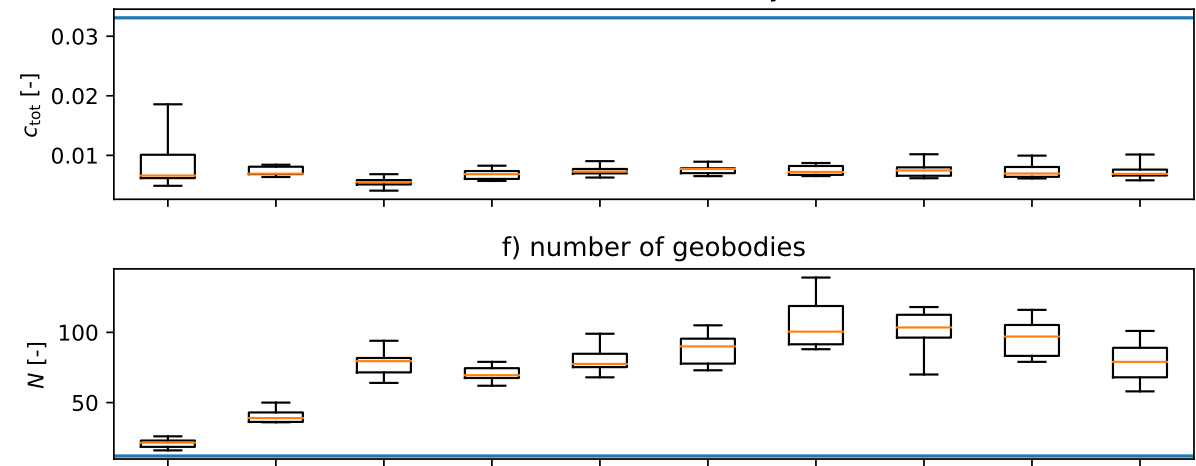

g) Jaccard index

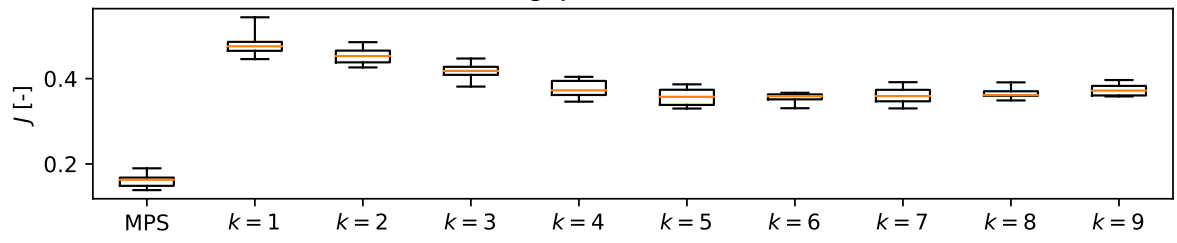

Figure 9: Comparison of the pure MPS and the hybrid approach. The latter was performed using $h^{\text {(true) }}$ as reference $h$, and $\left(\delta_{1}, \delta_{2}\right)=(0.01,0.001)$. The first boxplot on the left shows the results of 10 pure MPS simulations, while the subsequent boxplots, labeled with the index $k$, represent iterations performed with the hybrid approach. For each iteration, the results obtained from 10 hybrid simulations using a different random seed were aggregated 


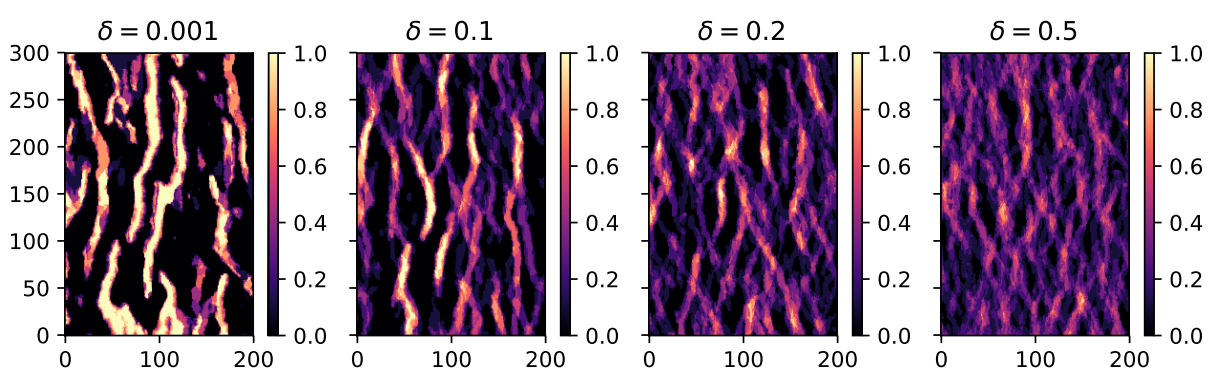

Figure 10: Mean value of simulations (facies codes) obtained over 10 iterations of hybrid approach with different thresholds

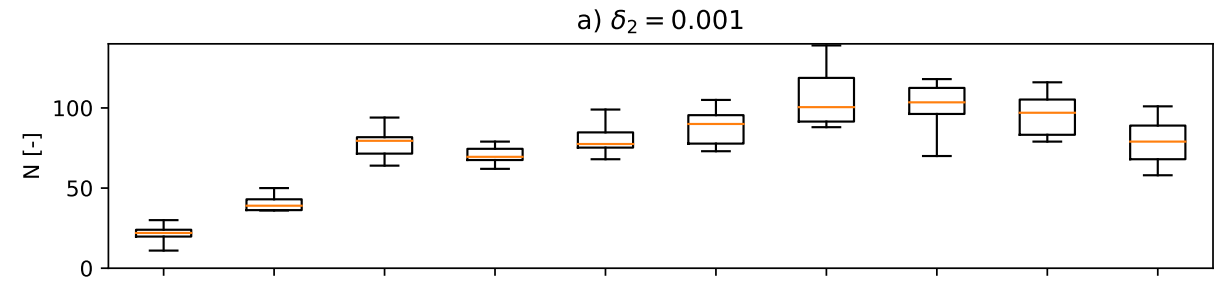

b) $\delta_{2}=0.1$

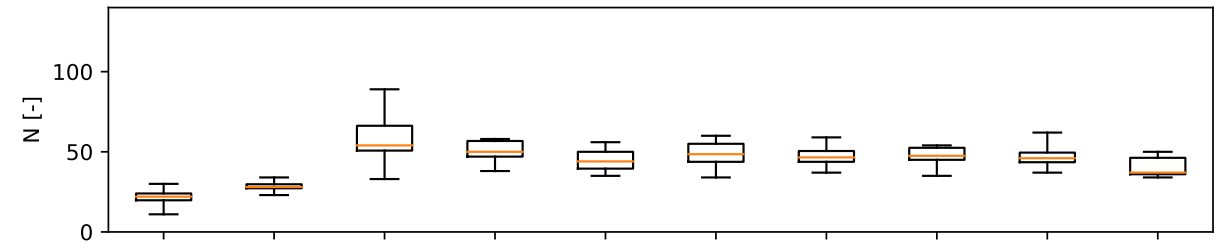

c) $\delta_{2}=0.2$

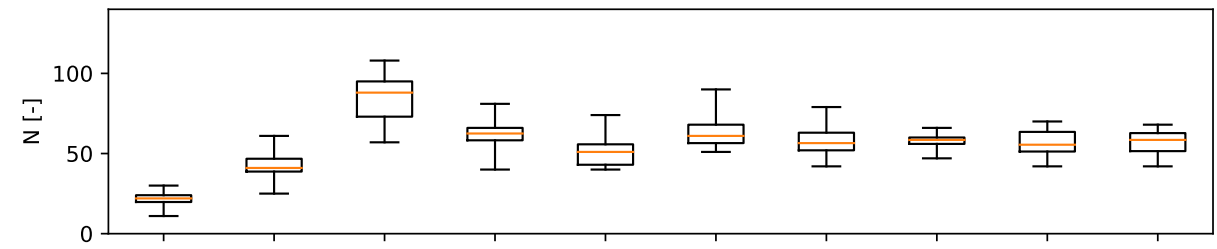

d) $\delta_{2}=0.5$

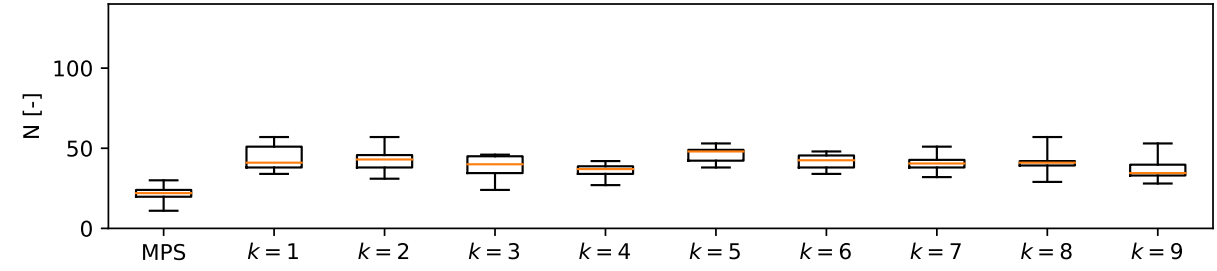

Figure 11: Boxplots of number of geobodies $N$ measured at each iteration for different values of the threshold $\delta$. The first boxplot corresponds to a pure MPS simulation 
a) $\delta_{2}=0.001$

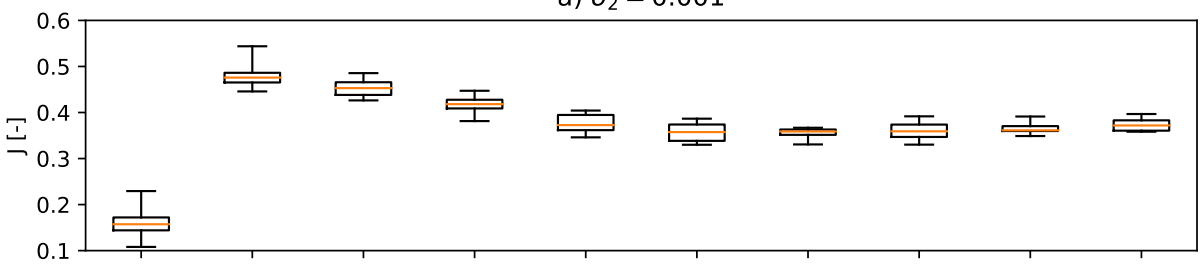

b) $\delta_{2}=0.1$
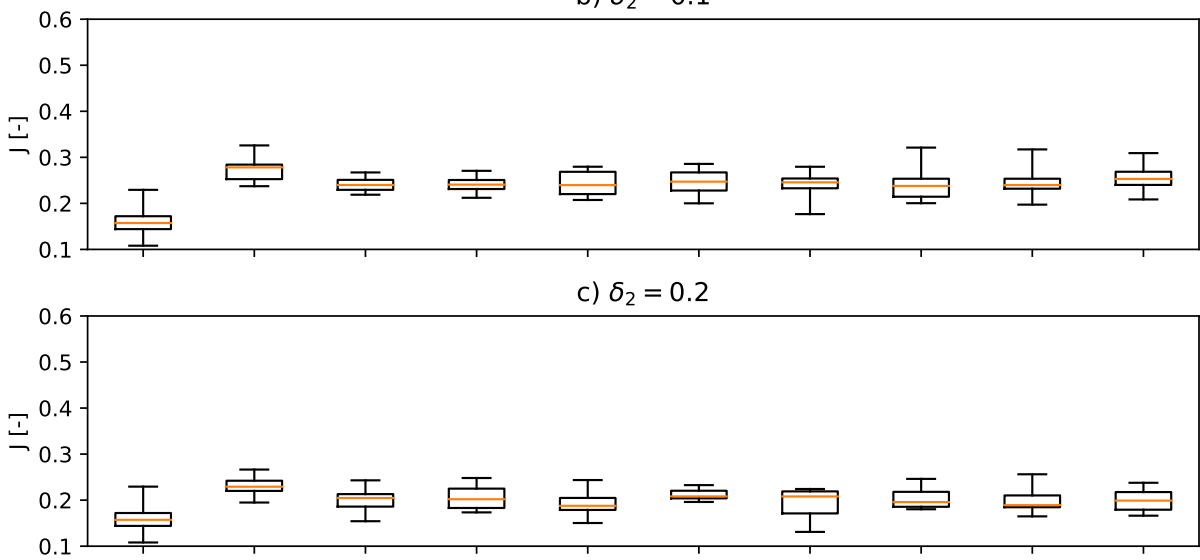

d) $\delta_{2}=0.5$

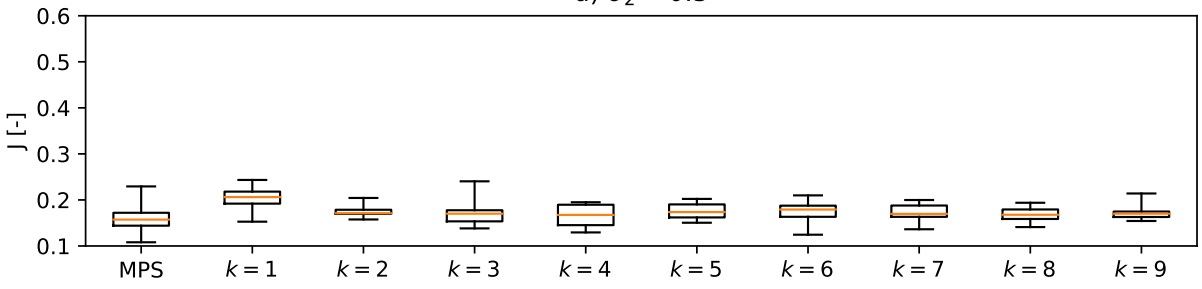

Figure 12: Boxplots of Jaccard index $J$ measured at each iteration for different values of the threshold $\delta$. The first boxplot corresponds to a pure MPS simulation 


\section{Discussion}

From the results obtained with the pure MPS approach (Fig. 5), it is clear that the range of variability of the considered parameters never includes the target value obtained for $T^{\text {(true) }}$. One could probably obtain better results using other parameter settings. Still, here the aim is not to look for the best parameterization of the MPS, but only to provide a reference case study to be compared with the hybrid approach, where the same parameters are used. Overall, the parameters computed on the subdomain show greater variability, which is probably due to the smaller number of cells on which the parameters are computed.

For the pure CMM approach, the parameters $|\lambda|, \lambda^{2}$, and $h^{(\mathrm{RMSE})}$ show an almost monotonic decreasing trend, confirming convergence of the maps $T^{(\mathrm{CM})}$ for increasing $k$ (Fig. 6). In particular, the parameter $\lambda^{2}$ attains a local minimum at the third iteration for the reduced domain. After this iteration, $\lambda^{2}$ for the whole domain and $|\lambda|$ show a slowing of their decreasing trend. The bias in the estimated $T$ field, which is quantified by $\lambda$, shows an approximately constant value up to the sixth iteration, then an increase for the reduced domain, whereas the increase of $\lambda$ over the whole domain shows a slight reduction after the third iteration. $h^{(\mathrm{RMSE})}$ at the third iteration has almost the same value as at the second iteration, showing a further decrease for subsequent iterations. These remarks suggest that continuing the iterations to $k>3$ does not significantly improve the results. This is supported by visual inspection of the maps of $T_{k}^{(\mathrm{CM})}$ obtained during the diverse iterations; here only the map obtained for the third iteration is shown (Fig. 7). Only the parameter $\lambda$ shows a discordant trend, which can be somehow explained by the fact that it is computed by mixing overestimates and underestimates of $T$, and therefore provides less clear information about the iterative procedure. The maps presented in Fig. 7, and in particular Fig. 7e, show that, in the case when $h^{(\text {ref })}=h^{\text {(true) }}$ the CMM reproduces the envelope of the true $T$ with good detail. Nevertheless, it is important to note that (i) the provided estimate in not categorical like the target $T$, (ii) there are strong differences between the percolating channels, i.e., the high-transmissivity channels that link the upper and lower part of the domain where the fixed head BCs are applied, and the disconnected channels, and (iii) noticeable differences in the estimated $T$ can be observed at the boundaries of the domain too.

The observations made for the pure CMM approach can be partially extended to the results of the hybrid approach. In this case, the same contrast between percolating channels and disconnected channels observed for the pure CMM approach in Fig. 7e can be observed for the secondary variable of the TI (Fig. 8h; see, for example, the almost shaded percolating channel that starts from the lower side of the domain close to the 150th cell). Again, the same contrast can be observed between $T^{\text {(est) }}$ close to the null flux boundary condition or close to the fixed head ones. All these observations suggest poor correlation between the primary variable and the secondary variable maps. The result of this poor correlation is evident in the final result (Fig. 8i), where the fit between the estimated channels and the true channels (Fig. 8d) is satisfactory only in the center of the domain. Nevertheless, the Jaccard index $J$ (and the parameters $p_{\text {rgt }}$ and $p_{\mathrm{wrg}}$ ) show that, on average, the hybrid method correctly guesses the position of the channels (Fig. 9b, c, g). While the proportions $p$ fluctuate slightly 
around the target value through the iterations, the connectivity indicators $\left(c_{\text {tot }}\right.$ and $\left.c_{\text {int }}\right)$ remain stable and comparable to the values estimated using the pure MPS approach. However, the boxplots of these latter indicators show smaller fluctuations, which are probably due to the additional constraints imposed by $h^{\text {(ref) }}$ in the hybrid approach. Overall, the number of geobodies is always bigger for the hybrid approach, indicating that, especially for $k>1$, the hybrid simulations are more fragmented than the pure MPS simulations. Figure 9 also shows that the first couple of iterations are decisive to fix the values of the statistics that have been examined while subsequent iterations introduce relatively small and often nonsystematic variations.

Concerning the sensitivity analysis performed on $\delta_{2}$, the stacks performed over the iterations clearly illustrate the importance of this parameter (Fig. 10). For small values $\left(0.001<\delta_{2}<0.1\right)$ the weight given to the information coming from $h^{(\mathrm{ref})}$ is strong and there are small differences between the iterations. When $\delta_{2}$ is increased, more freedom is left to the DS algorithm for the simulation of the categorical facies, and there is greater variability from one iteration to another. These observations are confirmed, for example, by the variability of $J$ and $N$ (Figs. 11 and 12). In the case of $N$, on average its values are smaller and closer to the pure MPS results. In other words, when the constraint on $h^{(\mathrm{ref})}$ is looser, the multivariate MPS produces more realistic channels. On the other hand, in terms of $J$, a stronger constraint on $h^{(\mathrm{ref})}\left(\delta_{2}<0.1\right)$ results in better localization of the channel facies and background facies in the simulation grid.

\section{Conclusions}

This work represents one of the first attempts to combine multiple-point statistics and a direct inversion method (the comparison model method, CMM) into a hybrid inversion approach. The results obtained so far are encouraging. The proposed hybrid approach allows one to address a number of limitations of the CMM: (i) it allows one to obtain multiple realizations of the estimated $T$ field by exploiting the stochasticity of the MPS; (ii) the result can be obtained directly as categorical variables; (iii) in the central part of the simulation domain, a good portion of the facies codes are estimated at the correct locations; (iv) some fine-scale structures can be partially reproduced. At the same time, the hybrid approach allows one to integrate auxiliary information about the hydraulic heads into multiple-point statistics.

In addition to these advantages, the results highlight a number of limitations of the proposed approach. First is the fact that the relationship between the primary variable and the secondary variable obtained with the CMM is not straightforward and can be misleading. Indeed, the latter variable is strongly dependent on the hydraulic gradient, and this results in very different responses; See, for example, the strong differences between percolating and disconnected channels (Fig. 8e, h). For the same reason, the correlation between the facies codes and the secondary variable computed by the CMM depends on the presence of boundary conditions.

To cope with these nonstationarities in the map of the secondary variable, one could incorporate into the MPS step additional maps representing, for example, the distance from the sides of the domain. A study of this setting deserves further investigation. 
The present study suffers from another limitation in that only a setting where $h^{\text {(ref) }}$ is fully known at all nodes of the simulation domain has been explored so far. Therefore, the next steps of this research should investigate the response of the hybrid approach to an input $h^{(\mathrm{ref})}$ obtained by interpolation of a limited number of measurements.

Another interesting result arising from the sensitivity analysis is the relevance of the threshold value for the secondary variable: smaller values (below 0.01 ) provide a good constraint on the observed hydraulic head, but deteriorate the overall properties provided by the first component of the TI. Therefore, for small values of the threshold the simulations appear more fragmented and less connected.

When combining two different tools such as multiple-point statistics and a direct inversion method, the number of simulation parameters grows to become somehow cumbersome. This is particularly true when the multivariate training image has more than two components, or the direct method is applied with a tomographic approach. These remarks should be recalled when generalizing the results of the test case discussed herein: the optimal choice of the parameters of the hybrid approach and of the two basic algorithms is not straightforward. Notwithstanding this growing complexity, the proposed hybrid approach opens new perspectives for inversion of hydraulic parameters of aquifers.

\section{Acknowledgements}

The authors thank P. Renard, G. Mariethoz and G. Pirot for the fruitful discussions, and two anonymous reviewers and the editor for their constructive comments, and J. Straubhaar and the University of Neuchâtel for providing the deesse simulation code.

\section{References}

Alcolea A, Renard P (2010) Blocking moving window algorithm: Conditioning multiple-point simulations to hydrogeological data. Water Resour Res 46(8), URL http://dx.doi.org/10.1029/2009WR007943

Ashby S, Falgout R (1996) A parallel multigrid preconditioned conjugate gradient algorithm for groundwater flow simulations. Nuclear Science Engineering 124(1):145-159

Carrera J, Neuman SP (1986) Estimation of aquifer parameters under transient and steady state conditions: 1. maximum likelihood method incorporating prior information. Water Resources Research 22(2):199-210, DOI 10.1029/ WR022i002p00199

Cattaneo L, Comunian A, de Filippis G, Giudici M, Vassena C (2015) Modeling groundwater flow in heterogeneous porous media with yagmod. Computation 4(1):2, DOI 10.3390/computation4010002

Comunian A, Renard P (2009) Introducing wwhypda: a world-wide collaborative hydrogeological parameters database. Hydrogeology Journal 17(2):481489, DOI 10.1007/s10040-008-0387-x 
De Filippis G, Giudici M, Margiotta S, Negri S (2016) Conceptualization and characterization of a coastal multi-layered aquifer system in the taranto gulf (southern italy). Environmental Earth Sciences 75(8):686, DOI 10.1007/ s12665-016-5507-7

de Marsily G, Lavedan G, Boucher M, Fasanino G (1984) Interpretation of interference tests in a well field using geostatistical techniques to fit the permeability distribution in a reservoir model. In: Verly Gea (ed) Geostatistics for natural resources characterization, Proceedings of the NATO Advanced Study Institute, Reidel Publishing Company, Dordrecht, p 831-849

Giudici M, Vassena C (2008) Spectral analysis of the balance equation of ground water hydrology. Transport in Porous Media 72(2):171178, DOI 10.1007/s11242-007-9142-3, URL http://dx.doi.org/10.1007/ s11242-007-9142-3

Guardiano FB, Srivastava RM (1993) Multivariate geostatistics: Beyond bivariate moments. In: Soares A (ed) Geostatistics: Troia '92, Kluwer, Dordrecht, The Netherlands, vol 1, pp 133-144

Gómez-Hernández JJ, Sahuquillo A, Capilla JE (1997) Stochastic simulation of transmissivity fields conditional to both transmissivity and piezometric data - i. theory. Journal of Hydrology 203(1):162 - 174, DOI http://dx.doi. org/10.1016/S0022-1694(97)00098-X

Jones JE, Woodward CS (2001) Newton-krylov-multigrid solvers for large-scale, highly heterogeneous, variably saturated flow problems. Advances in Water Resources 24(7):763 - 774, DOI 10.1016/S0309-1708(00)00075-0, URL http : //www.sciencedirect.com/science/article/pii/S0309170800000750

Kerrou J, Renard P, Franssen HJH, Lunati I (2008) Issues in characterizing heterogeneity and connectivity in non-multigaussian media. Advances in Water Resources 31(1):147 - 159, DOI http://dx.doi.org/10.1016/j.advwatres.2007. 07.002

Kollet J, Maxwell R (2006) Integrated surface-groundwater flow modeling: a free-surface overland flow boundary condition in a parallel groundwater flow model. Advances in Water Resources 29(7):945-958

Laloy E, Linde N, Jacques D, Mariethoz G (2016) Merging parallel tempering with sequential geostatistical resampling for improved posterior exploration of high-dimensional subsurface categorical fields. Advances in Water Resources 90:57 - 69, DOI http://dx.doi.org/10.1016/j.advwatres.2016.02.008

Lesnic D (2010) The comparison model method for determining the flexural rigidity of a beam. Journal of Inverse and Ill-posed Problems 18(5):577-590, DOI 10.1515/jiip.2010.026

Li L, Zhou H, Hendricks Franssen HJ, Gómez-Hernández JJ (2012) Groundwater flow inverse modeling in non-multigaussian media: performance assessment of the normal-score ensemble kalman filter. Hydrology and Earth System Sciences 16(2):573-590, DOI 10.5194/hess-16-573-2012 
Li L, Srinivasan S, Zhou H, Gómez-Hernández J (2013) A pilot point guided pattern matching approach to integrate dynamic data into geological modeling. Advances in Water Resources 62, Part A:125 - 138, DOI http://dx.doi. org/10.1016/j.advwatres.2013.10.008

Li L, Srinivasan S, Zhou H, Gomez-Hernandez J (2014) Simultaneous estimation of geologic and reservoir state variables within an ensemble-based multiplepoint statistic framework. Mathematical Geosciences 46(5):597-623, DOI $10.1007 / \mathrm{s} 11004-013-9504-\mathrm{z}$

Linde N, Renard P, Mukerji T, Caers J (2015) Geological realism in hydrogeological and geophysical inverse modeling: A review. Advances in Water Resources 86, Part A:86 - 101, DOI http://dx.doi.org/10.1016/j.advwatres.2015.09.019

Lochbühler T, Pirot G, Straubhaar J, Linde N (2014) Conditioning of multiplepoint statistics facies simulations to tomographic images. Mathematical Geosciences 46(5):625-645, DOI 10.1007/s11004-013-9484-z

Mariethoz G, Caers J (2014) Multiple-point Geostatistics: Stochastic Modeling with Training Images. Wiley, 376 pages

Mariethoz G, Renard P, Straubhaar J (2010) The direct sampling method to perform multiple-point geostatistical simulations. Water Resour Res 46(11):W11,536, DOI 10.1029/2008WR007621

Maxwell RM (2013) A terrain-following grid transform and preconditioner for parallel, large-scale, integrated hydrologic modeling. Advances in Water Resources 53:109 - 117, DOI https://doi.org/10.1016/ j.advwatres.2012.10.001, URL http://www.sciencedirect.com/science/ article/pii/S0309170812002564

Meerschman E, Pirot G, Mariethoz G, Straubhaar J, Meirvenne MV, Renard P (2013) A practical guide to performing multiple-point statistical simulations with the direct sampling algorithm. Computers \& Geosciences 52(0):307 324, DOI 10.1016/j.cageo.2012.09.019

Ponzini G, Crosta G (1988) The comparison model method: A new arithmetic approach to the discrete inverse problem of groundwater hydrology. Transport in Porous Media 3(4):415-436, DOI 10.1007/BF00233178

Ponzini G, Lozej A (1982) Identification of aquifer transmissivities: The comparison model method. Water Resources Research 18(3):597-622, DOI 10.1029/WR018i003p00597

Renard P, Allard D (2013) Connectivity metrics for subsurface flow and transport. Advances in Water Resources 51(0):168 - 196, DOI 10.1016/j.advwatres. 2011.12.001

Ronayne MJ, Gorelick SM, Caers J (2008) Identifying discrete geologic structures that produce anomalous hydraulic response: An inverse modeling approach. Water Resources Research 44(8):n/a-n/a, DOI 10.1029/ 2007WR006635, w08426 
Sahuquillo A, Capilla J, Gómez-Hernández J, Andreu J (1992) Conditional simulation of transmissivity fields honoring piezometric data. Hydraulic engineering software IV, Fluid flow modeling 2:201-214

Scarascia S, Ponzini G (1972) An approximate solution of the inverse problem in hydraulics. L'Energia Elettrica 49:518-531

Straubhaar J, Renard P, Mariethoz G, Froidevaux R, Besson O (2011) An improved parallel multiple-point algorithm using a list approach. Mathematical Geosciences 43(3):305-328, DOI 10.1007/s11004-011-9328-7

Strebelle S (2002) Conditional simulation of complex geological structures using multiple-point statistics. Mathematical Geology 34:1-21, DOI 10.1023/A: 1014009426274

Vassena C, Cattaneo L, Giudici M (2010) Assessment of the role of facies heterogeneity at the fine scale by numerical transport experiments and connectivity indicators. Hydrogeology Journal 18(3):651-668, DOI 10.1007/ s10040-009-0523-2

Vassena C, Rienzner M, Ponzini G, Giudici M, Gandolfi C, Durante C, Agostani D (2012) Modeling water resources of a highly irrigated alluvial plain (italy): calibrating soil and groundwater models. Hydrogeology Journal 20(3):449 467, DOI 10.1007/s10040-011-0822-2

Zhou H, Gómez-Hernández JJ, Li L (2014) Inverse methods in hydrogeology: Evolution and recent trends. Advances in Water Resources 63(0):22 - 37, DOI http://dx.doi.org/10.1016/j.advwatres.2013.10.014

Zinn B, Harvey CF (2003) When good statistical models of aquifer heterogeneity go bad: A comparison of flow, dispersion, and mass transfer in connected and multivariate gaussian hydraulic conductivity fields. Water Resour Res 39(3) 